\title{
High Cost/High Risk Components to Chalcogenide Molded Lens Model: Molding Preforms and Mold Technology
}

\author{
BE Bernacki
}

October 2012

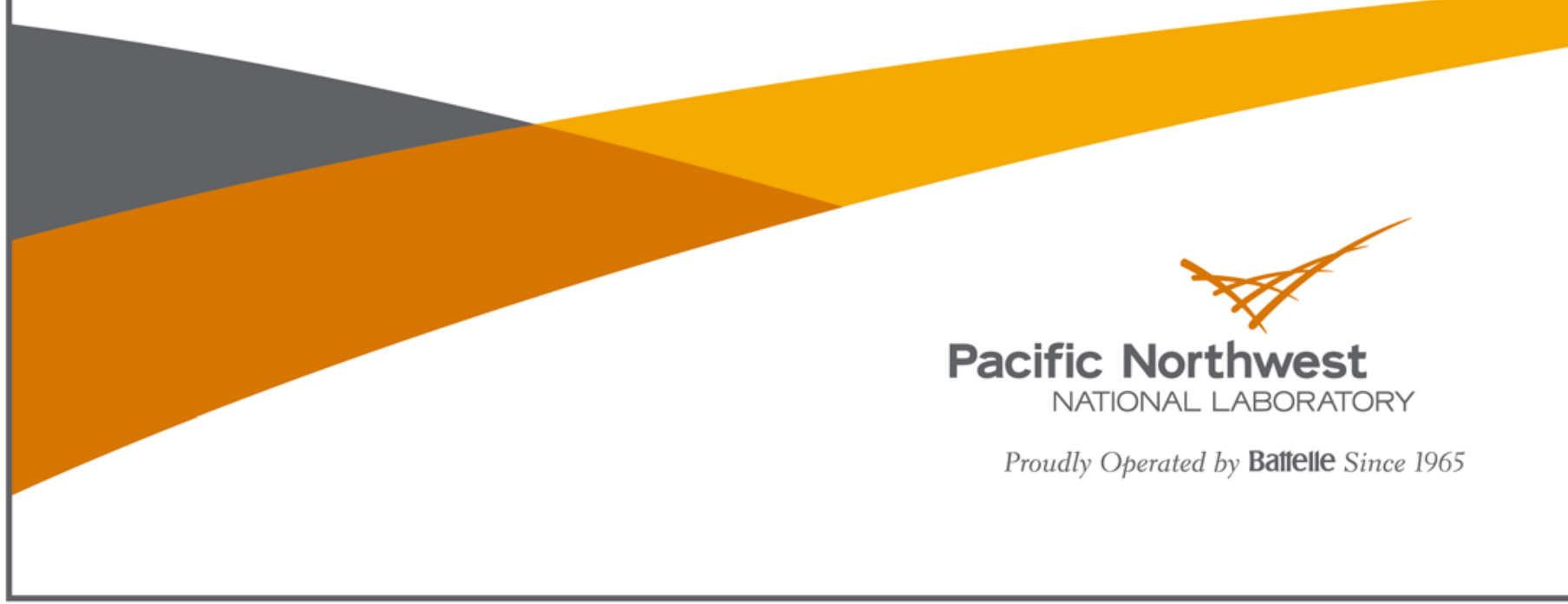




\title{
DISCLAIMER
}

This report was prepared as an account of work sponsored by an agency of the United States Government. Neither the United States Government nor any agency thereof, nor Battelle Memorial Institute, nor any of their employees, makes any warranty, express or implied, or assumes any legal liability or responsibility for the accuracy, completeness, or usefulness of any information, apparatus, product, or process disclosed, or represents that its use would not infringe privately owned rights. Reference herein to any specific commercial product, process, or service by trade name, trademark, manufacturer, or otherwise does not necessarily constitute or imply its endorsement, recommendation, or favoring by the United States Government or any agency thereof, or Battelle Memorial Institute. The views and opinions of authors expressed herein do not necessarily state or reflect those of the United States Government or any agency thereof.

\author{
PACIFIC NORTHWEST NATIONAL LABORATORY \\ operated by \\ BATTELLE \\ for the \\ UNITED STATES DEPARTMENT OF ENERGY \\ under Contract DE-AC05-76RL01830
}

Printed in the United States of America

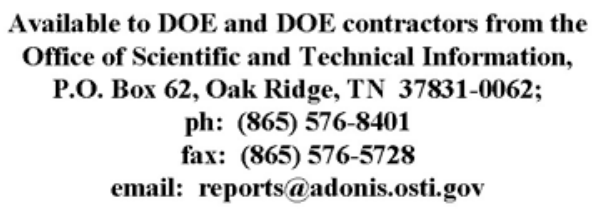

Available to the public from the National Technical Information Service 5301 Shawnee Rd., Alexandria, VA 22312 ph: (800) 553-NTIS (6847) email: orders@ntis.gov $<$ http://www.ntis.gov/about/form.aspx > Online ordering: http://www.ntis.gov 


\section{High Cost/High Risk Components to Chalcogenide Molded Lens Model: Molding Preforms and Mold Technology}

BE Bernacki

October 2012

Prepared for

the U.S. Department of Energy

under Contract DE-AC05-76RL01830

Pacific Northwest National Laboratory

Richland, Washington 99352 



\title{
High Cost/High Risk Components to Chalcogenide Molded Lens Model: Molding Preforms and Mold Technology
}

\author{
Bruce E. Bernacki, Ph.D. \\ Senior Research Scientist \\ Pacific Northwest National Laboratory, P. O. Box 999, Richland, WA 99352 \\ bruce.bernacki@pnnl.gov \\ 509-375-2135
}

\section{Introduction}

FiveFocal outlined the major components to a cost model for isothermal compression molding of chalcogenide lenses in view of alternative methods of fabrication, such as single point diamond turning (SPDT) or conventional grinding and polishing. There is certainly a threshold for lens production volume that self-selects molding over SPDT or grinding/polishing, and it is the aim of the FiveFocal model to determine this inflection point. Of the many components of the cost model, two main items are those concerning lens preforms and molding tooling technology. These two areas are the focus of the remainder of this document.

\section{Chalcogenide Glass Preforms}

A basic schematic for glass compression molding was outlined in the FiveFocal cost model document. Briefly, the approach to molding involves placing a volume of glass (or preform) between two mold halves that are shaped like the desired element surfaces and that are made to run coaxial with respect to each other, and then heating the preform/mold system to a temperature slightly above the glass transition temperature, $T_{g}$, where under control of the press controller, the velocity, force and temperature profiles the mold halves are driven together using an actuator to form the part.

\section{Preform Volume: Press to Final Dimension or Allow Post Processing?}

There are two main methodologies on molding and preform design. The first allows for post processing of the part after molding and requires that one have at least enough glass in the preform (or more) to mold the part to the desired volume. After molding, the part can be edge ground ("edged") to meet the specified outer diameter (OD). The second approach is to ensure (within production tolerances) that exactly enough glass is contained in the preform and by using a special mold design, to mold the part to the specified OD (within tolerance) with no post processing. The second method has many merits but it puts greater demands on the precision of preform production that will invariably affect yield, and hence, cost. However, using the second method allows for the molding within a mounting ring (for example, Alps and LightPath) which can then be bolted, soldered or YAG welded into the final optical assembly. An analysis needs to be undertaken that examines the cost of finishing the part by edge grinding versus higher preform costs of press-to-final-dimension approach as part of a cost model to determine each approach's relative merits. 


\section{Preform Shape}

The candidate design described by FiveFocal was that of a meniscus singlet with aspheric surfaces, as well as the option for a diffractive feature for achromatization on one surface. The meniscus shape is a natural consequence of the high index of the chalcogenide glass that drives the design to this "best form" shape to minimize the primary aberrations, notably spherical aberration. Early workers in molded glass lens technology thought that a near-net shape preform was necessary, probably to reduce the amount of glass that had to be moved to minimize stress and strain in the molded part. Eventually, the preform shape evolved to that of a sphere since there is substantial infrastructure to make ball lenses in high volume while maintaining tight dimensional tolerances, and hence, volume of the preform at reasonable cost. For visible molding glasses with the appropriate material characteristics, gob glass can be produced in which a precise volume of molten glass is metered out from a nozzle and allowed to drop through a fluid to cool and harden. Gobs are not spherical but are more closely hemispherically shaped. This is (to the best of the author's knowledge) not available for chalcogenide glasses as they must be melted in an oxygen-free sealed rocking furnace constructed of fused silica. Gob production would require similar restrictions on avoiding exposure of the molten glass to oxygen (a major impurity that introduces unwanted absorption) and probably does not exist beyond the laboratory (if at all). There is the use of the word "gob" in combination with chalcogenide glass lens molding in at least one recent paper ${ }^{1}$ but it is not known if this is literal and the authors have a process for chalcogenide gob production or whether they are simply using the term as a synonym for preform.

One desirable goal is to limit the amount of glass that must be moved during the press cycle to reduce subsequent stress and strain in the part, and to reduce the duration of the press cycle. Since the meniscus shape is the best form shape for chalcogenide singlets in general, and except for high numerical aperture lenses needed for quantum cascade laser $(\mathrm{QCL})$ collimation the sag is often not too great, the disk preform has great merit, if they can be produced economically, and is preferred over ball preforms.

Ball preforms have the advantage in that they can self-center in the concave mold half, but the convex mold half will contact the ball surface over a very small area and exert extremely high forces per unit area which may subject the preform to unwanted stress and strain. Also, although non-germanium glasses are relatively cheap (for IR glasses), they are not free and the production of ball preforms wastes glass which presently is not recycled as cull glass for chalcogenide glasses. The boule must be sliced, cored, tumbled to knock off the edges, rough ground, and then fine ground and polished to final dimensions to produce a ball lens or ball preform.

The near net shape preform might also have merit in that it would require the minimum amount of glass movement, and perhaps not significantly increase preform costs in sufficient quantities if processed off shore. Even diamond turning production houses obtain their blanks shaped to best fit spherical surfaces to limit machine time and tool wear, as SPDT machining passes are meant to remove only microns per

\footnotetext{
${ }^{1}$ D.H. Cha, H-J Kim, Y. Hwang, J.C. Jeong, and J-H Kim, "Fabrication of molded chalcogenide-glass lens for thermal imaging applications," Appl. Opt., 51, 5649-5656 (2012). 
pass and are not suited to machining the finished lens surface from a block of raw material. One idea that may have some merit is to obtain near net shaped preforms (essentially finished plano convex or meniscus lenses) that have a useful optical function without any further processing. For example, one could obtain plano convex lenses in chalcogenide materials suitable for simple tasks such as fiber coupling, basic imaging, laser focusing or collimation of low numerical aperture infrared fibers. These devices could be subjected to molding post processing to aspherize the first surface, emboss diffractive features, and mold the second surface, if required. If one has access to aspherical surface terms for one surface, the second concave spherical surface may be dispensed with in some circumstances, subject to tolerance analysis and requirements of the application.

\section{Preform Surface Quality}

In general, the finished molded part displays the surface roughness of the glass preform provided the mold has similar or better roughness characteristics. However, it has been observed by the author that some glasses can produce finished parts with surface roughness smoother than the starting preform since during the molding process the glass is smoothed by pressing against a much smoother mold surface. This is somewhat analogous to paper calendering, where paper is pressed between rollers under high pressure to produce the desired finished surface finish. It is unknown whether chalcogenide glasses can display this behavior, and if so, which of the various stable glass types currently commercially available might display this behavior. The biggest benefit would occur if one could use preforms that were fine ground and unpolished and combine both figure molding and surface finishing in the molding operation. This may be an area worthy of some investigation.

Chalcogenide glasses are also used to make photonic devices such as waveguides and modulators and to construct these devices thin films must be deposited using either thermal or laser-assisted deposition methods. One option to explore might involve depositing thin films of the same bulk chalcogenide material (to ensure coefficient of thermal expansion (CTE) matching) on molded optics to improve their surface finish if the coatings could be applied thick enough to planarize and smooth the surface.

Although at PNNL we have been limited to thermally evaporated chalcogenide thin films less than $5 \mu \mathrm{m}$ thickness this is due to CTE mismatches between the glass and the silicon substrate in waveguide construction. The large CTE mismatch between substrate and glass think film causes film delamination at greater film thicknesses, but should not be an issue if the substrate is CTE-matched. Finally, since these glasses absorb strongly in the visible and near IR, it may be possible to fire polish the surface using a high power visible or near-IR laser beam to locally reflow the chalcogenide glass as has been demonstrated with silica glasses, although I am unaware of anyone attempting this with infrared glasses. ${ }^{2}$

\footnotetext{
${ }^{2}$ K.M. Nowak, H.J. Baker, and D.R. Hall, "Efficient laser polishing of silica micro-optic components," Appl. Opt., 45, 162-171 (2006).
} 


\section{Preform Recycling}

It is unlikely that any molding process will have $100 \%$ yields, and the practical yield of a manufacturing process will influence the true cost of the product. Crystalline IR optical materials (germanium) are routinely recycled which results in two benefits: 1 ) recovery of some cost of the materials machined from the lens blanks, and 2) safe disposal of hazardous waste. Chalcogenide glasses are not recycled since strict control is necessary of the glass stoichiometry to ensure consistent glass melts for the different glass varieties. Some cost recovery might be possible if rejected parts (prior to anti reflection coating) could be remanufactured into preforms for re-use in the molding process.

\section{Mold Technology}

Materials known to be used in mold technology for glass lens pressing are lumped into two main categories: metals and ceramics. Both can be produced using deterministic methods. In the case of metals with ductile surfaces, SPDT is used, and numerically-controlled grinding is employed for ceramics. Both essentially use the same base machine (from companies such as Precitech ${ }^{3}$ or Moore $^{4}$ ) with either a diamond tool or grinding attachments. In general, ceramic molds offer greater lifetime with higher upfront costs to produce them. We will briefly look at both methods and examine their benefits and drawbacks.

\section{Metal Molds}

Historically, lens molding began with metal molds. In addition to spherical or aspherical surfaces, metal molds can produce diffractive surfaces easily using SPDT and special tools, and new slow and fast tool servo options on diamond turning machines offer the capability to directly turn lens array molds for new and novel IR applications not readily possible using a grinding approach, such as biconic or "potato chip" surfaces.

Corning based their technology on Inconel mold blanks, which are near net shaped (best-fit sphere) to the design, and then a thickness of electroless nickel is grown on the metal surface to provide a ductile material for SPDT. Metal molds can produce diffractive surfaces easily using SPDT and special tools, and new slow and fast tool servo options on diamond turning machines offer the capability to directly turn lens array molds for new and novel IR applications not readily possible using a grinding approach. The Inconel is heat treated to increase hardness after machining, as is the nickel optical surface after SPDT. Otherwise, the nickel would deform and crack or dent under molding pressure. The issue to avoid is to not anneal the Inconel during the heat treating of the nickel surface, which can be heat treated to hardness similar to that of hard chrome. Most alloys of aluminum can be diamond turned to excellent surface finish, and might be candidates for short run or soft tooling applications as there may be alloys of aluminum that may be suitable for chalcogenide lens molding due to the relatively low $T_{g}$ of

${ }^{3}$ http://www.precitech.com/

${ }^{4}$ http://www.nanotechsys.com/ 
chalcogenide glasses. Free machining brass can be used as soft tooling for higher temperature visible glass molding, but mold life is poor and it is only suitable for short, proof-of-concept runs. However, a good surface finish is possible with this material using SPDT. Rapid prototyping may provide some soft tooling or short run options since metals may now be laser sintered to tolerances as small as $\pm 40 \mu \mathrm{m}$ in materials such as Inconel 718. Whether optical-type surfaces would be amenable to this approach is unknown. Metal molds are necessary (at least one mold half, anyway) for diffractive or hybrid lenses, since the diffractive provide requires the use of small half-radius tools to machine the tiny grooves necessary to cause diffraction. There is a paper describing machining of diffractive surfaces using specialized grinding tools, but there is no evidence they could be applied to the curved convex surface in the example hybrid design. ${ }^{5}$ Furthermore, it appears they require specially-shaped grinding tools that may not provide a general or economical process for mold production.

\section{Ceramic Molds}

Ceramic molds are typically one of the commercial varieties of tungsten carbide (WC) which can be rough machined using wire EDM and finished using deterministic grinding. Larger mold radii are shaped using a rotating grinding wheel on a spindle with the rotation of the tool orthogonal to the part rotation on the machine spindle. Smaller parts (this may not be an issue for chalcogenide lenses except perhaps for those needed for small quantum cascade laser applications) are ground using a stick grinder rotating at an angle around $45^{\circ}$ with respect to the spindle axis.

\section{Mold Coatings}

Mold coatings such is TiN or TiN/SiC can be used for mold protection and mold release, as well as one of the commercially-available diamond like carbon (DLC) coatings for either mold technology. In general, the major categories of coatings can be divided into ceramic nitride coatings, noble metal coatings, and carbon-based coatings (DLC). However, true DLC coating must be applied at high temperatures, and so depending on the metallic mold material, one of the DLC-like coatings in which hydrogen is substituted for some of the carbons may have to be employed, although these restrictions are not required with ceramic molds due to their high temperature characteristics. A recent article provides a complete survey of available coatings and methodology for testing and selecting the appropriate coating solution. ${ }^{6}$

\section{Summary and Recommendations}

Two main areas of an isothermal lens molding cost model were examined: lens preforms and mold technology (materials and coatings). More attention was paid to the preform topic as the author has more extensive first-hand experience in this area, while mold technology tends to be proprietary and practitioners avoid teaching their competition through academic articles or patent filings.

\footnotetext{
${ }^{5}$ H. Suzuki, T. Moriwaki, Y. Yamagata, and T. Higuchi, “Precision machining and measusrement of micro aspheric molds," Proc. ISUPEN2011 (2011).

${ }^{6}$ F. Klocke, K. Georgiadis, O. Dambon, K.D. Bouzakis, S. Gerardis, G. Skordaris, “Complete qualification methodology for coatings of precision glass molding tools," Opt. Eng., 51, 073401-1 - 073401-9 (2012).
} 
Cost-effective preform production is essential to a successful molding program. Visible molding glasses are available in either gob or ball format. Chalcogenide glasses must be melted in an oxygen-free environment in order to prevent impurities that result in absorption features in the glasses' spectral transmission. A gob approach ought to be the goal of any long term chalcogenide molding program. For a small company, partnering with a university professor such as Kathleen Richardson of CREOL (formerly of Clemson) at the University of Central Florida would be the most productive route to explore preform production involving gob methods. As for a currently-available approach to preforms, I see only disk preforms being practical due to potentially reduced material waste and perhaps the ability to also save costs by either not pressing to final OD (to increase yield due to less restrictive control on preform volume) and finishing to final OD by edging or by relaxing preform surface roughness specifications and using the molds and pressing process to improve the surface quality of the as-produced preforms in the finished part as described in the text, if this proves feasible.

As for mold technology, metal mold technology is the most accessible technology for a start-up or small company with limited resources but there is an aspect of know-how in the growth of electroless nickel to obtain smooth, hard surfaces without delamination from the mold base metal. Metal molds allow almost any shape to be mastered and replicated using more mature SPDT technology and the new, state-of-the-art 5-axis machines, including the mastering of lens arrays using a fast tool servo, and also hold out the hope of rapid delivery using either soft tooling allows (aluminum or free machining brass) or exploiting laser sintering rapid prototyping technologies. Ceramic molds by far offer longer mold lifetimes but with higher up-front costs and slower turn-around on mold fabrication and refurbishing. Also, many types of surfaces may not be possible to master due to the relatively large tool radius of the grinding tools combined with the fact that tool wear is much greater during the fabrication process and thus requires greater control and knowledge to maintain the desired surface figure. Although WC is the most commonly mentioned ceramic mold material in the literature, there are many commercial varieties and selection of the optimum type is essential enjoy acceptable mold quality with few "pull outs" after grinding in the finished surface. Also, although not explored in the text, there may be casting methods to produce the roughed out ceramic molds ready for final machining that could reduce turn around and cost of ceramic molds. To get more insight into commercial state-of-the-art, working with another university researcher, Allan Yi of Ohio State may provide more concrete information to help choose one or the other approach. ${ }^{7}$ Insight into state-of-the-art deterministic machining processes can be explored by working with the Precision Engineering Center at North Carolina State University. ${ }^{8}$

Mold coatings are also closely held secrets, but some approaches are mentioned in the literature, for example, TiN, Pt-Ir, or the varieties of DLC coatings. The danger here is that many of these coating are used across industries with requirements quite different from optical fabrication. For example, there are many TiN processes commercially available but it gets more challenging to find one that can provide a thin conformal coating that won't distort the mold figure you spent so much time and money to

\footnotetext{
${ }^{7}$ http://www.ise.osu.edu/ISEFaculty/yi/PERL-OSU.pdf

${ }^{8}$ http://www.pec.ncsu.edu/
} 
produce with acceptable surface roughness. Here, I would find a vendor that provides the process and rely on them to get you up to speed unless you have a coating guru on staff.

Although not strictly the topic of this focused evaluation, one must not ignore the need and cost associated with metrology. Metrology is needed to confirm mold figure, surface roughness of the mold, finished parts and preforms, and performance of the finished part, ideally using transmission interferometry. Otherwise some type of probe metrology is needed such as the Talysurf. ${ }^{9}$ Once the process is under control, lenses can be sampled but $100 \%$ inspection should be carried out for optical performance and cosmetic defects.

Finally, one ignores the existing and evolving SPDT technology for directly fabricating lenses from a variety of IR materials at their peril. The process is well understood and forgiving, and can be automated using pick and place robots to share operators between multiple machines, reduce labor costs and increase production throughput. Excellent 2-axis SPDT machines can be had in the $\$ 200 \mathrm{~K}$ range, and keeping several of these running three shifts a day can produce a lot of product before molding would be viable or the much desired "killer app" materializes.

${ }^{9}$ http://www.taylor-hobson.com/optics-pgi.html 


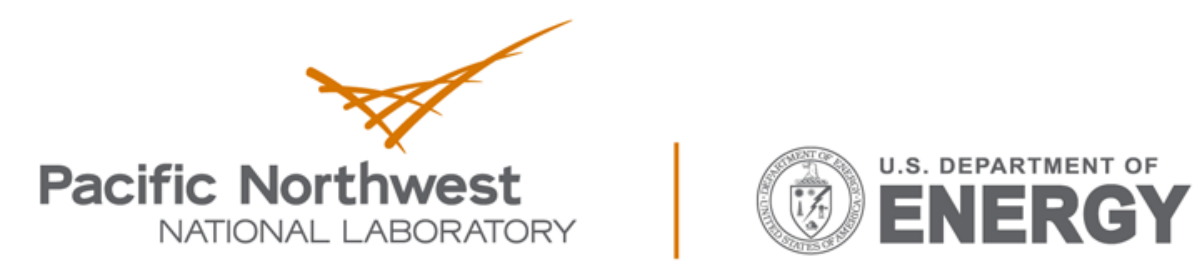

Proudly Operated by Battelle Since 1965

902 Battelle Boulevard

P.O. Box 999

Richland, WA 99352

1-888-375-PNNL (7665)

www.pnnl.gov 